\title{
Evaluation and Characterization of Cowpea (Vigna unguiculata L. Walp) Genotypes for Growth, Yield and Quality parameters in Prayagraj Agro Climatic Region
}

\author{
Shyam Sundar Dangi ${ }^{1 *}$, Bineeta M. Bara ${ }^{2}$, A.K. Chaurasia ${ }^{2}$ and Abhishek Kumar Pal ${ }^{1}$ \\ ${ }^{1}$ Seed Science and Technology, ${ }^{2}$ Department of Genetics and Plant Breeding, \\ Naini Agricultural Institute, Sam Higginbottom University of Agriculture, Technology and \\ Sciences, Prayagraj, 211007 U. P., India
}

*Corresponding author

\begin{tabular}{l} 
K e y w o r d s \\
Cowpea (Vigna \\
unguiculata \\
L.Walp), \\
Characterization, \\
Quality parameters, \\
Vigour, Nodulation \\
and seed yielding \\
attributes \\
Article Info \\
\hline $\begin{array}{l}\text { Accepted: } \\
24 \text { September } 2020 \\
\text { Available Online: } \\
10 \text { October } 2020\end{array}$ \\
\hline
\end{tabular}

\section{A B S T R A C T}

The experiment evaluation and characterization of cowpea (Vigna unguiculata L.Walp) genotypes for growth, yield and quality parameters in Prayagraj agro-climatic region was conducted at Field Experimental centre as well as in post graduate Seed Testing Laboratory, Department of Genetics and Plant Breeding, Sam Higginbottom University of Agriculture, Technology and Sciences, Prayagraj (U.P.). India during Kharif season 2019. in order to standardize the best genotype of Cowpea. Thirteen genotype of cowpea $\left(\mathrm{G}_{1}-\right.$ CP-1, G 2 - CP-3, G $3-$ CP-5, G 4 - CP-7, G - CP-10, G 6 - CP-11, G - CP-12, G - CP$\left.13, \mathrm{G}_{9}-\mathrm{CP}-14, \mathrm{G}_{10}-\mathrm{CP}-15, \mathrm{G}_{11}-\mathrm{CP}-16, \mathrm{G}_{12}-\mathrm{CP}-17, \mathrm{G}_{13}-\mathrm{PL}-4\right)$ were evaluated by screening Seed yielding attributes viz., field parameters include field emergence, days to $50 \%$ flowering, days to maturity, plant height, number of pods per plant, number of seeds per pod, number of nodules per plant, nodules fresh weight, seed yield per plant, seed yield per plot, biological yield and harvest index and different seed quality parameters viz., germination percentage, root length, shoot length, seedling length, seedling fresh weight, seedling dry weight, seedling vigour index-I, seedling vigour index-II, 100 seed weight. It was found that all the genotype showed significance difference with lowest value $\left(\mathrm{G}_{4}-\right.$ CP-7) and the highest seedling length, seedling fresh weight, seedling dry weight; vigour index and yielding attributes were observed for $\mathrm{G}_{3}-\mathrm{CP}-5$. Highest germination was observed in $\mathrm{G}_{11}-\mathrm{CP}-16$ and $\mathrm{G}_{3}-\mathrm{CP}-5$.

\section{Introduction}

Cowpea (Vigna unguiculata L. Walp) belongs to the family Fabaceae and having chromosome number $2 \mathrm{n}=22$ with genus
Vigna. Cowpea is an important leguminous crop is believed to be originated in Central Africa. It is self-pollinated annual herb with a wide range of growth habit and response to photoperiod. In country, it is cultivated 
mainly in Gujarat, West Bengal, Tamil Nadu, Andhra Pradesh, Kerala and Orissa. In Gujarat, it is mainly grow in Sabarkantha, Banaskantha, Mehasana, Patan, Ahmedabad, Kheda and Anand district and commonly known as "chowli" in this area. In India, the total area under beans cultivation is about 136 (M ha) with the production 1373 (MT) (Anonymous, 2018-2019).

In India, Cowpea is well adapted to arid and semi-arid areas due to its morphological as well as biochemical characteristics. The deep rooted system and its short duration life cycle are some of the factors that make cowpea very adaptable to hostile environments. Cowpea is adapted to high temperatures (20$35^{\circ} \mathrm{C}$ ) and grows well in a wide range of soil texture, from heave clays, if well drained, to sandy; it grows best in slightly acid to alkaline soils ( $\mathrm{pH} 5.5-6.5$ ). Cowpea grows under wide extreme of moisture condition and once established it is fairly drought tolerant (Gaiser and Graef, 2001). It is often grown in rain-fed agriculture receiving at least $600 \mathrm{~mm}$ annual rainfall (Valenzuela \& Smith, 2002).It requires very few inputs, as the plants root nodules are able to fix atmospheric nitrogen, making it a valuable crop for resource poor farmers and well-suited to intercropping with other crops. The whole plant is used as forage for animals, with its use as cattle feed likely responsible for its name.

Cowpea (Vigna unguiculata L.Walp) is an important legume widely cultivated in tropics and subtropics for forage, green pods and grains (Ali et al., 2004). Cowpea is usually better adapted to drought, high temperature and other biotic stresses compared with other crops (Kuykendall et al., 2000; Martins et al., 2003). Protein content of cowpea seed is among the highest in cultivated legumes (Aremu et al., 2007) and can serve as an excellent source of dietary protein in animal feeds. The mature grain contains 20 to $25 \%$ of protein, 1.3 to $1.5 \%$ lipid and 5.1 to $5.8 \%$ crude fiber (Tshovhote et al., 2003).

Number of superior cowpea varieties are released by different states, universities and ICAR institutes but no study has been carried out with respect to suitability of specific variety of cowpea for certain region. So, there is urgent need to evaluate the cowpea varieties released from states and national levels and made a certain recommendation to generate research evidences of different varieties with respect to their suitability under certain conditions to benefits the cowpea growers of Allahabad.

\section{Materials and Methods}

The experiment was conducted at Field Experimental centre as well as in post graduate Seed Testing Laboratory, Department of Genetics and Plant Breeding, Sam Higginbottom University of Agriculture, Technology and Sciences, Prayagraj (U.P.). In order to standardize the best genotype of Cowpea, thirteen genotype of cowpea $\left(\mathrm{G}_{1}-\right.$ $\mathrm{CP}-1, \mathrm{G}_{2}-\mathrm{CP}-3, \mathrm{G}_{3}-\mathrm{CP}-5, \mathrm{G}_{4}-\mathrm{CP}-7, \mathrm{G}_{5}-$ $\mathrm{CP}-10, \mathrm{G}_{6}-\mathrm{CP}-11, \mathrm{G}_{7}-\mathrm{CP}-12, \mathrm{G}_{8}-\mathrm{CP}-13$, $\mathrm{G}_{9}-\mathrm{CP}-14, \mathrm{G}_{10}-\mathrm{CP}-15, \mathrm{G}_{11}-\mathrm{CP}-16, \mathrm{G}_{12}-$ $\left.\mathrm{CP}-17, \mathrm{G}_{13}-\mathrm{PL}-4\right)$ are used as experiment to evaluate best genotype in prayagraj agro climatic condition for growth yield and seed quality parameters. After cleaning and grading, the seeds are placed on between paper method in laboratory and line method in field used for sowing.

The observation on the characters viz.

\section{Field parameters}

Field emergence (Kotowski, 1926), days to $50 \%$ flowering, days to maturity, plant height, no. of pods per plant, no. of seeds per pod, no. of nodules per plant, nodules fresh weight (Khan, 2006), seed yield per plant, seed yield per plot, biological yield and harvest index. 


\section{Quality parameters}

Germination percent (ISTA, 2004), Root length $(\mathrm{cm})$, Shoot length $(\mathrm{cm})$, Seedling length $(\mathrm{cm})$, Seedling Fresh weight $(\mathrm{g})$, Seedling dry weight (g), Seedling Vigour index length, Seedling Vigor index mass (Baki and Anderson, 1973)and 100 seed weight were recorded. The experimental data recorded were subjected to statistical analysis for calculating analysis of variance, range, mean, critical difference and coefficient of variation for RBD design Panse and Sukhatme (1967) and for CRD design (Fisher, 1936).

\section{In field}

\section{Field emergence (\%)}

One hundred seeds from each treatment in three replications will be used for field emergence studies. The seeds will be sown in well prepared at $3 \mathrm{~m}$ deep. The field emergence count will be taken on the $4^{\text {th }}, 7^{\text {th }}$ and $10^{\text {th }}$ day after sowing and the emergence percentage will be calculated taking into account the number of seedlings emerged three centimeter above the soil surface (Kotowski, 1926).

Field emergence $(\%)=$

Total Number of seedling emerged

Total no. of seeds sown

\section{Days to $50 \%$ flowering}

The numbers of days were count till the days of $50 \%$ flower initiation from the date of sowing.

\section{Days to maturity}

The numbers of days from plant sowing to plant harvest (physiological maturity) were count manually from each genotype.

\section{Plant height (cm)}

It was measured from ground level to the base of the top most fully opened leaf at harvesting stage. Average height of five plants was recorded in centimeters.

\section{Number of pods per plant}

The total numbers of pods from five randomly selected plants were counted manually from each genotype.

\section{Number of seeds per pod}

The total numbers of seeds from five randomly selected plant pods were counted manually from each genotype and divided by total number of pods.

\section{Number of nodules per plant}

Five plants from each genotype plot in three replication will be uprooted 30 days after seedling (DAS), and the extent of nodulation were estimated by carefully washing the roots and detaching the nodules before counting (Khan, 2006).

\section{Nodules fresh weight (g)}

After washing the root nodules from the field the nodules were detached from the plant roots and weighed in an electronic weigh balance for fresh weight of root nodules expressed in grams $(\mathrm{g})$.

\section{Seed yield per plant (g)}

The seed weights of five randomly select plants were recorded of each plot.

\section{Seed yield per plot (g)}

The seed weight of total plants in a plot was recorded. 


\section{Biological yield}

The biological yield refers to the total dry matter accumulation of a plant system. The biological yield of five randomly select plants were recorded of each plot.

\section{Harvest index}

For grain crops, harvest index (HI) is the ratio of harvested grain to total shoot dry matter, and this can be used as a measure of reproductive efficiency. The HI of five randomly select plants were recorded of each plot.

Grain yield
Harvest index $(\%)=$
$\begin{gathered}\text { Biological yield } \\ \text { Biold }\end{gathered}$

\section{In Laboratory}

Germination per cent: For taking observation regarding seed germination. 100 seeds were kept blotting paper (BP method). The paper folds were kept in germination in an upright position at constant temperature $25^{\circ} \mathrm{C} \pm 22^{\circ} \mathrm{C}$ and $80 \% \mathrm{RH}$. Three replications were made for each treatment with First count was taken on $4^{\text {th }}$ day and Final count made after 7 days of treatment.

Root, shoot, and seedling length: For recording seedling length, 10 seedlings from each sample were taken randomly. The length of roots, shoots and total seedling were measured. To find out the average length of roots, shoots and total seedling of the sample, mean often seedlings were taken.

Seedling fresh weight: For recording seedling fresh weight, 10 seedlings from each sample were taken randomly. The fresh weight of seedlings was weighted on electronic balance.
Seedling dry weight: For taking the observation of seedling dry weight, 10 seedlings were dried $100^{\circ} \mathrm{C}$ temperatures for 1 hours. The dried seedlings were weighted on electronic balance.

Seedling vigour index length: Seedling vigor index was calculated by adopting the method suggested by Abdul Baki and Anderson (1973).

Seedling vigor index length $=$ Germination (\%) x Total seedling length $(\mathrm{cm}$.)

Seedling vigour index mass: Vigor index in terms of mass is determined by the multiplication of germination percentage with seedling dry weight on the day of final count.

Seedling vigor index mass= germination $(\%)$ $\mathrm{X}$ seedling dry weight

100 seed weight: Count 100 seeds by manually or electric seed counter from each variety and weighing on electronic balance.

\section{Results and Discussion}

According to the results, all studied traits were evaluated on their characters and there was completely significant difference between highest value and lowest value of cowpea seeds.

All growth and yield attributes viz., field emergence, days to $50 \%$ flowering, days to maturity, plant height $(\mathrm{cm})$, no. of pods per plant, no. of seeds per pod, no. of nodules per plant, nodules fresh weight $(\mathrm{g})$, seed yield per plant $(\mathrm{g})$, seed yield per plot $(\mathrm{g})$, biological yield $(\mathrm{g})$ and harvest index $(\%)$ were observed by $\mathrm{G}_{3}-\mathrm{CP}-5$ significantly recorded maximum where found in lowest $\left(\mathrm{G}_{4}-\mathrm{CP}-7\right)$ (Table- 1$)$.

All seedling characters viz. Germination percent, Root length $(\mathrm{cm})$, Shoot length $(\mathrm{cm})$, 
Seedling length $(\mathrm{cm})$, seedling fresh weight (g), seedling dry weight (g), Seedling Vigour index length, Seedling Vigor index mass and 100 seed weight were observed by $\mathrm{G}_{3}-\mathrm{CP}$ 5significantly recorded maximum where found in lowest $\left(\mathrm{G}_{4}-\mathrm{CP}-7\right)$ (Table- 2$)$.

Significantly maximum percentage of field emergence $(89.00 \%)$ was recorded by $\mathrm{G}_{11}$ $\mathrm{CP}-16$ and it was followed by $\mathrm{G}_{3}-\mathrm{CP}-$ $5(87.00 \%)$, Minimum field emergence percentage was recorded by $\mathrm{G}_{4}-\mathrm{CP}-$ $7(78.00 \%)$.Similar results of field emergence percent was observed by Agbogidi and Egho (2011); Teame et al.,(2017)and Gbaguidi et al.,(2013).

Minimum days to $50 \%$ flowering(32.66)was recorded by $\mathrm{G}_{8}-\mathrm{CP}-13$ and it was followed by $\mathrm{G}_{12}-\mathrm{CP}-17(32.66)$, Maximum days to $50 \%$ flowering were recorded by $\mathrm{G}_{6}-\mathrm{CP}-$ 11(62.66).Similar results of days to $50 \%$ flowering was observed by Ekpo et al.,(2012); Naim et al.,(2011); Dadson et al., (2003)and Muhammad et al., (1994).

Minimum days to maturity(72.00)was recorded by $\mathrm{G}_{3}-\mathrm{CP}-5$ and it was followed by $\mathrm{G}_{11}-\mathrm{CP}-16(72.33)$, Maximum days to maturity were recorded by $\mathrm{G}_{10}-\mathrm{CP}$ 15(84.66).Similar results of days to maturity was observed by Basavaraj et al., (2013); Padulosil and Ng (1997); Ibrahima et al., (2013)and Sharma et al., (2015).

Maximum plant height at harvesting stage $(117.46 \mathrm{~cm})$ was recorded by $\mathrm{G}_{9}-\mathrm{CP}$ 14and it was followed by $\mathrm{G}_{11}-\mathrm{CP}-16(103.13$ $\mathrm{cm})$, Minimum plant height was recorded by $\mathrm{G}_{2}-\mathrm{CP}-3(67.50 \mathrm{~cm})$.Similar results of plant height was observed by Vural and Karasu (2007); Khan et al., (2010) and Pasqueth (1998).

Maximum number of pods per plant(13.46)was recorded by $\mathrm{G}_{3}-\mathrm{CP}-5$ and it was followed by $\mathrm{G}_{11}-\mathrm{CP}-16(13.33)$, Minimum number of pods per plant was recorded by $\mathrm{G}_{4}-\mathrm{CP}-7$ (5.80). Similar results of number of pods per plant was observed by Fageria et al., (2011); Peksen (2004) and Babaji et al., (2011).

Maximum number of seeds per pod(16.13)was recorded by $\mathrm{G}_{3}-\mathrm{CP}-5$ and it was followed by $\mathrm{G}_{10^{-}}$CP-15(14.60), Minimum number of seeds per pod was recorded by $\mathrm{G}_{4}-\mathrm{CP}-7(9.85)$.Similar results of number of seeds per pod was observed by Gulumser et al., (1989); Sunil Kumar et al., (2015)and Sharma et al., (2013).

Maximum number of nodules per plant(46.66)was recorded by $\mathrm{G}_{11}-\mathrm{CP}-16$ and it was followed by $\mathrm{G}_{8}-\mathrm{CP}$ 13(44.60), Minimum number of nodules per plant was recorded by $\mathrm{G}_{7}-\mathrm{CP}-$ 12(23.43).Similar results of number of nodules per plant was observed by Kuykendall et al., (2000); Philipand Watters et al., (1991)and Harris,(2014).

Maximum nodules fresh weight per plant(0.852 gm)was recorded by $\mathrm{G}_{11}-\mathrm{CP}-16$ and it was followed by $\mathrm{G}_{3}-\mathrm{CP}-7(0.650$ gm),Minimum nodules fresh weight per plant was recorded by $\mathrm{G}_{7}-\mathrm{CP}-12(0.203$ gm).Similar results of nodules fresh weight was observed by George et al., (2014); Mehta et al., (2010); Finch-Savage (1993) and Sarmadi et al., (2014).

Significantly higher seed yield per plant and per plot of different cowpea genotypes (18.55 and $1613.90 \mathrm{~g}$ ) was recorded by $\mathrm{G}_{3}-\mathrm{CP}-5$ and it was followed by $\mathrm{G}_{11}-\mathrm{CP}-16$ (16.29 and $1295.80 \mathrm{~g}$ ) respectively. Minimum seed yield per plant was recorded by $\mathrm{G}_{4}-\mathrm{CP}-7$ (9.09 and $615.80 \mathrm{~g}$ ) respectively. Similar results of seed yield was observed by Basaran et al., (2011); Alhaji Hamza Ibrahim, (2008); Singh et al., (2007) and Quaye et al., (2011). 
Maximum biological yield (4636.94 gm) was recorded by $\mathrm{G}_{3}-\mathrm{CP}-5$ and it was followed by $\mathrm{G}_{11}-$ CP-16 (3800.01 gm), Minimum biological yield was recorded by $\mathrm{G}_{4}-\mathrm{CP}$ $7(2689.21 \mathrm{gm})$. Similar results of biological yield was observed by Shiringani and Shimeles (2011) and Ali et al., (2004); Brahim et al., (2006).

Maximum harvest index (34.80\%) was recorded by $\mathrm{G}_{3}-\mathrm{CP}-5$ and it was followed by $\mathrm{G}_{11}-\mathrm{CP}-16(34.10 \%)$, Minimum harvest index was recorded by $\mathrm{G}_{4}-\mathrm{CP}-7(22.90 \%)$.Similar results of harvest index was observed by Singh (1997); Peksen and Art1k (2004) and Chattopadhyay et al., (2014).

Significantly higher percentage of germination $(92.00 \%)$ was recorded by $\mathrm{G}_{11}-$ CP-16 and it was followed by $\mathrm{G}_{3}-\mathrm{CP}-5$ (91.00\%), Minimum percentage of germination was recorded by $\mathrm{G}_{4}-\mathrm{CP}-7$ $(83.00 \%)$. Similar finding was reported by Peksen et al., (2004), Ndunguru and Summerfield (2005), Rojas et al., (2000).

Maximum root length (16.42 cm)was recorded by $\mathrm{G}_{11}-\mathrm{CP}-16$ and it was followed by $\mathrm{G}_{3}-\mathrm{CP}-5(15.40 \mathrm{~cm})$,Minimum root length was recorded by $\mathrm{G}_{4}-$ CP-7(10.10 $\mathrm{cm})$.Maximum shoot length $(21.02 \mathrm{~cm})$ was recorded by $\mathrm{G}_{3}-\mathrm{CP}-5$ and it was followed by $\mathrm{G}_{11}-\mathrm{CP}-16(19.42 \mathrm{~cm})$, Minimum shoot length was recorded by $\mathrm{G}_{4}-$ CP-7(13.97 $\mathrm{cm})$.Maximum length of seedling (36.42 $\mathrm{cm})$ was recorded by $\mathrm{G}_{3}-\mathrm{CP}-5$ and it was followed by $\mathrm{G}_{11}-\mathrm{CP}-16(35.84 \mathrm{~cm})$, Minimum seedling length was recorded by $\mathrm{G}_{4}-\mathrm{CP}-7(24.07 \mathrm{~cm})$.

Root length is usually a good indicator for seed vigour measurement which may contribute towards better establishment of seedling. Similar results were observed by Serraj et al., (2004), Rosenberg and Rinne (1986), Socioconsult (2006). Variations in shoot length for different genotypes might be due to the difference in genetic constitution of seeds. Such type of findings also reported by Sabaghpour et al., (2003), Roberts et al., (1988), Agrawal et al., (1990). Seedling length is good indicator of seed vigour which indicated towards better plant establishment. Similar findings were reported by Socioconsult (2006), Rojas et al., (2000).

Maximum seedling fresh weight (5.87 gm)was recorded by $\mathrm{G}_{3}-\mathrm{CP}-5$ and it was followed by $\mathrm{G}_{11}-\mathrm{CP}-16(5.42 \mathrm{gm})$, Minimum seedling fresh weight was recorded by $\mathrm{G}_{4-}$ CP-7(3.12 gm). Maximum seedling dry weight $(0.674 \mathrm{gm})$ was recorded by $\mathrm{G}_{3}-\mathrm{CP}-$ 5and it was followed by $\mathrm{G}_{11}-\mathrm{CP}-16(0.610$ $\mathrm{gm})$, Minimum seedling dry weight was recorded by $\mathrm{G}_{4}-\mathrm{CP}-7(0.416 \mathrm{gm})$. Seedling fresh weight had significant correlated with seedling length. With increase in length of a seedling, this seems a sharp rise in fresh weight. These results were agreement with the finding of Babu et al., (2010), Gawade (2008), Lev et al., (2000). Seedling dry weight had significantly positively correlated with seedling length and seedling fresh weight. Similar results were reported by Kaur et al., (2008), Lev et al., (2000) and Saraf et al., (1998).

Maximum seed vigor index length (3313.70) was recorded by $\mathrm{G}_{3}-\mathrm{CP}-5$ and it was followed by $\mathrm{G}_{11}-\mathrm{CP}-16$ (3297.95), Minimum seed vigor index length was recorded by $\mathrm{G}_{4}-\mathrm{CP}-7$ (1998.32). Results of present finding were reported by Shaibu and Ibrahim (2016), Rosenberg and Rinne (1986), Ndunguru and Summer field (2005).

Significantly maximum seed vigor index mass (61.35) was recorded by $\mathrm{G}_{3}-\mathrm{CP}-5$ and it was followed by $\mathrm{G}_{11}-\mathrm{CP}-16$ (56.16),Minimum seed vigor index mass was recorded by $\mathrm{G}_{4}-$ CP-7 (34.43). Results of present finding were reported by Shaban (2003), Igbal (2015), Jomova et al., (2005), Rojas et al., (2000). 
Table.1 Mean performance of 13 cowpea genotypes for 12 growth and yield characters

\begin{tabular}{|c|c|c|c|c|c|c|c|c|c|c|c|c|c|}
\hline S.NO & $\begin{array}{c}\text { Genotyp } \\
\text { es }\end{array}$ & $\begin{array}{c}\text { Field } \\
\text { Emergen } \\
\text { ce } \\
\text { percenta } \\
\text { ge } \\
\end{array}$ & $\begin{array}{c}\text { Days to } \\
\mathbf{5 0 \%} \\
\text { Flowerin } \\
\text { g }\end{array}$ & $\begin{array}{c}\text { Days to } \\
\text { Maturi } \\
\text { ty }\end{array}$ & $\begin{array}{c}\text { Plant } \\
\text { height } \\
(\mathrm{cm})\end{array}$ & $\begin{array}{c}\text { Numbe } \\
\text { r of } \\
\text { Pods } \\
\text { Per } \\
\text { plant }\end{array}$ & $\begin{array}{c}\text { Numbe } \\
\text { r of } \\
\text { Seeds } \\
\text { per } \\
\text { Pods } \\
\end{array}$ & $\begin{array}{l}\text { Number } \\
\text { of } \\
\text { Nodules } \\
\text { per plant }\end{array}$ & $\begin{array}{l}\text { Nodul } \\
\text { es } \\
\text { Fresh } \\
\text { weight } \\
\text { (g) }\end{array}$ & $\begin{array}{c}\text { Seed } \\
\text { yield } \\
\text { per } \\
\text { plant } \\
(\mathrm{g})\end{array}$ & $\begin{array}{c}\text { Seed yield } \\
\text { per plot } \\
\text { (g) }\end{array}$ & $\begin{array}{l}\text { Biologic } \\
\text { al yield } \\
(\mathrm{g})\end{array}$ & $\begin{array}{c}\text { Harvest } \\
\text { index }\end{array}$ \\
\hline 1 & $\mathrm{G}_{1}$ & 79 & 35.00 & 76.00 & 88.40 & 11.23 & 11.73 & 29.93 & 0.300 & 10.94 & 904.20 & 3391.59 & 26.66 \\
\hline 2 & $\mathrm{G}_{2}$ & 83 & 35.33 & 74.66 & 67.50 & 10.83 & 10.46 & 32.66 & 0.373 & 10.19 & 797.80 & 3264.63 & 24.44 \\
\hline 3 & $\mathrm{G}_{3}$ & 87 & 33.33 & 72.00 & 95.40 & 13.46 & 16.13 & 43.36 & 0.650 & 18.55 & 1613.90 & 4636.94 & 34.80 \\
\hline 4 & $\mathrm{G}_{4}$ & 78 & 35.66 & 77.66 & 78.93 & 5.80 & 9.85 & 28.53 & 0.213 & 9.09 & 615.80 & 2689.21 & 22.90 \\
\hline 5 & $\mathrm{G}_{5}$ & 84 & 35.33 & 75.33 & 83.70 & 6.13 & 10.90 & 34.86 & 0.483 & 11.89 & 1050.40 & 3453.24 & 30.42 \\
\hline 6 & $\mathrm{G}_{6}$ & 82 & 62.66 & 83.33 & 94.83 & 9.66 & 12.75 & 29.73 & 0.280 & 11.29 & 997.06 & 3461.11 & 28.81 \\
\hline 7 & $\mathrm{G}_{7}$ & 80 & 36.33 & 77.66 & 77.40 & 7.86 & 11.36 & 23.43 & 0.203 & 12.74 & 1120.70 & 3444.08 & 32.54 \\
\hline 8 & $\mathrm{G}_{8}$ & 86 & 32.66 & 73.66 & 89.26 & 12.16 & 13.93 & 44.60 & 0.513 & 13.49 & 1165.00 & 3467.29 & 33.60 \\
\hline 9 & $\mathrm{G}_{9}$ & 81 & 34.00 & 76.33 & 117.46 & 10.46 & 12.01 & 24.13 & 0.240 & 11.62 & 1010.20 & 3384.25 & 29.85 \\
\hline 10 & $\mathrm{G}_{10}$ & 84 & 61.66 & 84.66 & 80.50 & 11.66 & 14.60 & 32.80 & 0.386 & 12.08 & 1080.86 & 3404.45 & 31.75 \\
\hline 11 & $\mathrm{G}_{11}$ & 89 & 33.00 & 72.33 & 103.13 & 13.33 & 14.21 & 46.66 & 0.852 & 16.29 & 1295.80 & 3800.01 & 34.10 \\
\hline 12 & $\mathrm{G}_{12}$ & 83 & 32.66 & 74.00 & 79.60 & 8.33 & 13.53 & 33.40 & 0.413 & 9.86 & 802.33 & 2948.69 & 27.21 \\
\hline 13 & $\mathrm{G}_{13}$ & 85 & 36.33 & 76.33 & 74.80 & 9.00 & 11.62 & 32.13 & 0.320 & 11.32 & 960.51 & 3427.99 & 28.02 \\
\hline \multicolumn{2}{|c|}{ Grand Mean } & 83.15 & 38.77 & 76.46 & 87.00 & 10.00 & 12.55 & 33.56 & 0.402 & 12.26 & 1031.89 & 3444.12 & 29.62 \\
\hline \multicolumn{2}{|c|}{ C.D. $(5 \%)$} & 2.92 & 1.30 & 1.82 & 8.64 & 1.76 & 1.44 & 3.67 & 0.05 & 1.55 & 46.63 & 70.87 & 0.57 \\
\hline \multicolumn{2}{|c|}{ SE(m) } & 1.00 & 0.44 & 0.62 & 2.96 & 0.60 & 0.49 & 1.26 & 0.02 & 0.53 & 15.98 & 24.28 & 0.19 \\
\hline \multicolumn{2}{|c|}{$\operatorname{SE}(d)$} & 1.42 & 0.63 & 0.88 & 4.19 & 0.85 & 0.70 & 1.78 & 0.03 & 0.75 & 22.59 & 34.34 & 0.27 \\
\hline \multicolumn{2}{|c|}{ C.V. } & 2.09 & 1.98 & 1.41 & 5.89 & 10.46 & 6.83 & 6.49 & 7.69 & 7.51 & 2.68 & 1.22 & 1.14 \\
\hline
\end{tabular}


Table. 2 Mean performance of 13 cowpea genotypes for 9 seedlings attributes

\begin{tabular}{|c|c|c|c|c|c|c|c|c|c|c|}
\hline$\stackrel{\dot{0}}{\dot{Z}}$ & & : & 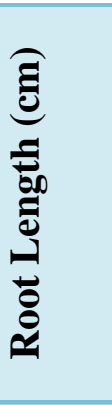 & 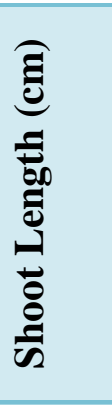 & 己 & 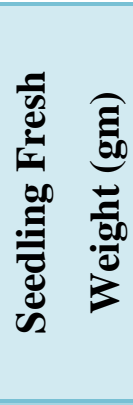 & 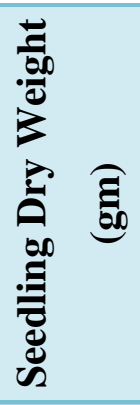 & 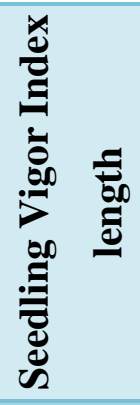 & 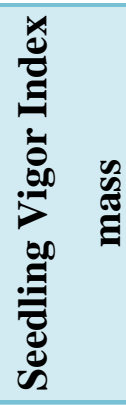 & 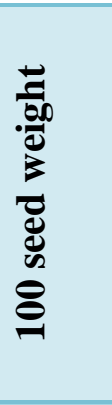 \\
\hline 1 & $\mathbf{G}_{1}$ & 84 & 10.50 & 15.09 & 25.59 & 3.12 & 0.434 & 2149.62 & 36.44 & 9.10 \\
\hline 2 & $\mathbf{G}_{2}$ & 87 & 11.85 & 16.34 & 28.19 & 4.15 & 0.481 & 2452.62 & 41.87 & 11.12 \\
\hline 3 & $\mathbf{G}_{3}$ & 91 & 15.40 & 21.02 & 36.42 & 5.87 & 0.674 & 3313.70 & 61.35 & 12.40 \\
\hline 4 & $\mathbf{G}_{4}$ & 83 & 10.10 & 13.97 & 24.07 & 3.35 & 0.416 & 1998.32 & 34.43 & 8.17 \\
\hline 5 & $\mathbf{G}_{5}$ & 88 & 12.75 & 16.02 & 28.77 & 4.68 & 0.502 & 2533.80 & 44.19 & 9.81 \\
\hline 6 & $\mathbf{G}_{6}$ & 86 & 11.84 & 15.62 & 27.46 & 3.95 & 0.466 & 2363.97 & 40.07 & 10.53 \\
\hline 7 & $\mathbf{G}_{7}$ & 84 & 10.95 & 14.36 & 25.31 & 3.51 & 0.441 & 2125.22 & 36.99 & 9.61 \\
\hline 8 & $\mathbf{G}_{8}$ & 89 & 14.45 & 18.56 & 33.01 & 4.94 & 0.570 & 2939.47 & 50.71 & 11.70 \\
\hline 9 & $\mathbf{G}_{9}$ & 85 & 11.17 & 16.10 & 27.27 & 4.45 & 0.508 & 2319.25 & 43.26 & 10.21 \\
\hline 10 & $\mathbf{G}_{10}$ & 89 & 13.42 & 17.03 & 30.45 & 4.58 & 0.537 & 2709.49 & 47.83 & 11.39 \\
\hline 11 & $\mathbf{G}_{11}$ & 92 & 16.42 & 19.42 & 35.84 & 5.42 & 0.610 & 3297.95 & 56.16 & 12.16 \\
\hline 12 & $\mathbf{G}_{12}$ & 86 & 11.52 & 15.37 & 26.89 & 3.69 & 0.460 & 2314.20 & 39.62 & 10.80 \\
\hline 13 & $\mathbf{G}_{13}$ & 90 & 14.10 & 17.60 & 31.70 & 5.07 & 0.547 & 2852.07 & 49.27 & 12.03 \\
\hline \multicolumn{2}{|c|}{$\begin{array}{l}\text { Grand } \\
\text { Mean }\end{array}$} & 87.23 & 12.65 & 16.65 & 29.30 & 4.36 & 0.511 & 2566.89 & 44.78 & 10.69 \\
\hline \multicolumn{2}{|c|}{ C.D. $(5 \%)$} & 2.69 & 0.93 & 1.63 & 2.02 & 0.53 & 0.07 & 195.05 & 5.92 & 0.40 \\
\hline \multicolumn{2}{|c|}{$\mathbf{S E}(\mathbf{m})$} & 0.94 & 0.32 & 0.57 & 0.70 & 0.18 & 0.02 & 68.34 & 2.07 & 0.14 \\
\hline \multicolumn{2}{|c|}{ C.V. } & 2.15 & 5.16 & 6.85 & 4.82 & 8.53 & 9.71 & 5.32 & 9.24 & 2.67 \\
\hline
\end{tabular}

Significantly higher 100 seed weight (12.40 gm) was recorded by $\mathrm{G}_{3}-\mathrm{CP}-5$ and it was followed by $\mathrm{G}_{11-}$ CP-16(12.16 gm),
Minimum 100 seed weight was recorded by $\mathrm{G}_{4}-\mathrm{CP}-7$ (8.17 gm). 100-seed weight was principal yield contributing traits. Similar 
results have been also reported by Henry et al., (2003), Verma et al., (2005), Dharmalingam et al., (1989), Stahr (2012).

In conclusion, the evaluation of genotypic performance of different genotype of cowpea, significantly in both field and lab conditions. Total 13genotypes check in this experiment and observe $\mathrm{G}_{3}(\mathrm{CP}-5)$ followed by $\mathrm{G}_{11}$ (CP16), and $\mathrm{G}_{13}$ (Pant lobia-4) significantly increased the growth, yield and quality parameters of cowpea. Among the all 13genotypes of cowpea CP-5 showed maximum quality and yielding characters and find out all characters lowest in $\mathrm{G}_{4}$ (CP-7). These conclusions are based on the results of six months investigation and therefore further investigation is needed to arrive at valid recommendations.

\section{References}

Agbogidi, O. M. and Egho, E.O. (2012). Evaluation of eight varieties of cowpea (Vigna unguiculata (L.)Walp) in Asaba agro-ecological environment, Delta State, Nigeria. Basic Research Journal of Agricultural Science and Review, 1: 6368.

Agrawal, R.L. and Panwar, Anil (1990). Identification of soybean varieties on the basis of seed and seedling characteristic. Seed Res., 18(1): 77-81.

AlhajiHamza Ibrahim. (2008). Yield Performance of Some Cowpea Varieties under Sole and Intercropping with Maize at Bauchi, Nigeria. An International Multidisciplinary Journal, Ethiopia. 2(3): 278-291.

Ali Y, Aslam Z, Hussain F, Shakur A (2004).Genotype and environmental interaction in cowpea (Vigna unguiculata$L$ Walp) for yield and disease resistance Inter. J. Environ. Sci. Technol., 1 (2): 119 123.

Anonymous (2014-15).Indian Horticulture Database - 2014.NHB, Ministry of Agriculture, Government of India.
Aremu C.O, Ariyo O.J, Adewale B.D (2007).Assessment of selection techniques in genotype $\mathrm{X}$ environment interaction in cowpea Vigna unguiculata (L.) walp. Afr. J. Agric. Res., 2 (8): 352-355.

Babu K.D,Patel R.K, Singh A, Yadav D.S, De L.C, Deka B.C (2010). Seed germination, seedling growth and vigour of papaya under North east Indian condition. ActaHorticulturae. 851:299-306.

Baki A, Anderson JD.(1973). Vigor determination in Soybean seed by multiple criteria. Crop Sci. 13: 630-633.

Basaran, Ugur.;Ayan, Ilknur.; Acar, Zeki.; Mut, Hanife and AsciOzlem, Onal (2011). Seed yield and agronomic parameters of cowpea (Vigna unguiculata LWalp.) genotypes grown in the Black Sea region of Turkey. Afr. J. Agri. Res. 10 (62): 13461-13464.

BasavarajMakanur, V. K. Deshpande S. K. and Vyakaranahal, B. S. (2013). Characterization of cowpea genotypes based on Quantitative descriptors, The Bioscan, 8 (4): 1183-1188.

Brahim, M. M, Rafiq, A., Sultan, M. and Goheer, M. A., (2006). Green fodder yield and quality evaluation of maize and cowpea sown alone and in combination. $J$. Agric. Res. 44: 15.

Chattopadhyay A, Rana N.P, Seth T, Das S, Chatterjee S, Dutta S. (2014). Identification of selection indices and choosing of parents for vegetable cowpea (Vigna unguiculata cvgr. sesquipedalis) breeding programme. Legume Research, 37(1): 19-25.

Dadson R.B, Hashem F.M, Javaid I, Joshi J and Allen A.L (2003).Response of Diverse Cowpea Genotypes to Drought. (CDROM) Annual Meeting Abstracts. ASA, CSSA, SSSA: Madison, WI.

Dharmalingam, V. and Kadamba Vanasundaram, M. (1989). Genetic divergence in Cowpea. Madras agric. J., 76 (7): 394- 399.

Ekpo, I. A., Agbor, R. B., Osuagwu, A. N., Okpako, E. C. and Ekanem, B. E. (2012).Evaluation of Eight Cowpea (Vigna unguiculata l. Walp). Int. J. Pure Appl. 
Sci. Technol. 12(2): 1-7.

Fageria N.K, Baligar V.C, Jones C.A (2011).Growth and Mineral Nutrition of Field Crops. CRC Press, Boca Raton.

Finch-Savage W. E., (1993). The effects of osmotic seed priming and the timing of water availability in the seed bed on the predictability of carrot seedling establishment in the field. Acta Horticulture, 267: 209-216.

Fisher, R.A. (1936). The correlation between relative on the supposition of genotypes grown in Kumaun Himalaya, Indian Journal Genetics. 66(1): 37-38.

Gaiser, T. and Graef, F. (2001). Optimization of a parametric land evaluation method for cowpea and mearl millet production in semiarid regions. Agronomic 21: 705-712.

Gawade U.S. (2008). Seed viability, germination and seedling growth studies in custard apple M.Sc. (Ag.)Thesis, Dr. Panjabrao Deshmukh Krishi Vidyapeeth, Akola MS, (INDIA).

Gbaguidi A.A, Dansi A, Loko L.Y, Dansi M, Sanni A (2013). Diversity and agronomic performances of the cowpea (Vigna unguiculata (L.) Walp.) landraces in Southern Benin. Intl. J. Agron. Plant Prod. 4(5):936-949.

George, W., Mmbaga., Kelvin, M., Mtei., Patrick, A. Ndalddemi., (2014). Extrapolations on the Use of Rhizobium Inoculants Supplemented with Phosphorus (P) and Potassium (K) on Growth and Nutrition of Legumes. Agricultural Sciences, 5: 1207-1226.

Harris, D., (2014). On-farm seed priming reduces risk and increases yield in tropical crops. Australian Agronomy Conference, 12(4).

Henry, A. and Mathur, B.K. (2003). Varietal divergence in Cowpea. In: Advances in arid legume research (Eds. A. Henry, D. Kumar and N. B. Singh) Sci. Publ., pp. 6770.

Ibrahima, Z. D.; Akromah, R. and James, Y. A. (2013). Comparative study of cowpea germplasm diversity from Ghana and mali using morphological characteristics. J.
Plant Breed. Genet. 1(03): 139-147.

Igbal M.A (2015). Improving Germination and Seedling Vigour of Cow pea (Vigna unguiculata L. Walp) with Different Techniques. American-Eurasian Journal of Agricultural and Environmental Sciences 15(2):265-270.

ISTA (2004).International Seed Testing Association (2004) International rules for seed testing. ISTA, Zürich: P. 206.

Jomova, K. Benkova, M., Zakova, M., Gregova, E. and Kraic, J. (2005). Clustering of chickpea (Cicer arietinum L.) accessions. Genetic Resources and Crop Evolution, 52: 1039-1048.

Kaur G., Kumar S., Nayyar H., Upadhyaya H. D. (2008). Cold stress injury during the pod-filling phase in Chickpea (Cicer arietinum L.): effects on quantitative and qualitative components of seeds. J. Agron. Crop Sci. 194 457-464.

Khan A, Bari A, Khan S, Hussain N.S, Zada I (2010). Performance of cowpea genotypes at higher altitude of NWFP Pak. J. Bot., 42(4): 2291-2296.

Khan, A.A. (2006).Preplant physiological seed conditioning. Horticultural Reviews, 14: 131,81 .

Kotowski, F. (1926). Temperature relations Rep. for period April 1963-March to germination of vegetable 1964. Submitted to U.S.D.A. 100 p. seeds. Proc. Amer. Sot. Hort. Sci. 23: 176-184.

Kuykendall L.D, Hashem F.M, Dadson R.B, Elkan G.K (2000).Nitrogen Fixation. In: Encyclopedia of Microbiology, Lederberg, J., (Ed.). Academic Press, New York pp: 329-404.

Lev-Yadun S., Gopher, A. and Abbo, S., (2000). The cradle of agriculture. Science 288: 1062-1063.

Martins L.M.V, Xavier G.R, Rangel F.W, Ribeiro J.R.A, Neves M.C.P, Morgado L.B, Rumjanek N.G (2003). Contribution of biological fixation to cowpea: A strategy for improving seed yield in the semi-arid region of Brazil. Biol. Fertil. Soils, 38: 333-339.

Mehta, D. IC, Kanwar, H. S., Thakur, A. K. and 
Thakur, K. S., (2010). Influence of organic seed priming on germination and seedling quality in bell pepper (Capsicum annuиm L.). Journal of Hill Agriculture, 1(1):85-87.

Muhammad G, Ramazan C.M, Aslam M and Chaudhry G.A (1994).Performance of cowpea cultivars under rainfed conditions. $J$ of Agric Res 32(1): 119-122.

Naim, M. E. Ahmed, Jabereldar, A. A. and Mohamed, E. A. (2011). Effect of seed rate and varieties on vegetative growth attributes of cowpea (Vigna unguiculataL.Walp) under rain-fed in Sudan. Asian Journal of Science and Technology. 2 (3) : 022-026.

Ndiaga, C. (2000).Genotype x Row Spacing and Environment interaction of cowpea in semi-arid zones. African Crop Science Journal. 9(2): 359-367.

Ndunguru B.J, Summerfield R.J (2005). Comparative laboratory studies of cow pea (Vigna unguiculata L.Walp) and soybean (Glycine max) under tropical temperature conditions. I. Germination and hypocotyls elongation. East African Agricultural and Forestry Journal 41:58-64.

Padulosil, S. and Ng, N. Q., (1997).Origin, taxonomy, and morphology of Vigna unguiculata (L.)Walp.". In Singh, B. B.; Mohan, D. R.; Dashiell, K. E.; Jackai, L. E. N. Advances in Cowpea Research (PDF). Ibadan, Nigeria: International Institute of Tropical Agriculture and Japan International Research Center for
Agricultural Sciences.

Panse V. G., and Sukhatme P. V. (1967). Statistical Methods for Agricultural Workers. Indian Council of Agricultural Research: Pp. 103-108.

Pasqueth RS (1998).Morphological study of cultivated cowpea Vigna unguiculata (L.) Walp. Importance of ovule number and definition of $\mathrm{cv}$ gr Melanophthalmus. Agronomie, 18: 61-70.

Peksen A, Peksen E, Bozoglu H (2004). Relationships among some seed traits, laboratory germination and field emergence in cow pea ((L.) Walp.) genotypes. Pakistan Journal of Botany 36(2):311-320.

Peksen E, Artık C (2004). Comparison of some cowpea (Vigna ungiuculata (L).Walp) genotypes from Turkey for seed yield and yield related characters. J. Agron. 3(2):137-140.

Philip, R. D. and Watters, M. C., (1991). Contribution of cowpea to nutrition and health. Food Technology 9: 127 - 130.

Quaye-Addo, A. A., Darkwa, A. A. and Ampiah, M. K. P. (2011). Performance of three cowpea (Vigna unguiculata (L) Walp) varieties in two agro-ecological zones of the central region of Ghana ii: grain yield and its components. $A R P N$ Journal of Agricultural and Biological Science.6 (2): 1-9.

\section{How to cite this article:}

Shyam Sundar Dangi, Bineeta M. Bara, A.K. Chaurasia and Abhishek Kumar Pal. 2020. Evaluation and Characterization of Cowpea (Vigna unguiculata L. Walp) Genotypes for Growth, Yield and Quality parameters in Prayagraj Agro Climatic Region. Int.J.Curr.Microbiol.App.Sci. 9(10): 3069-3079. doi: https://doi.org/10.20546/ijcmas.2020.910.369 